Tropical Journal of Pharmaceutical Research May 2020; 19 (5): 949-955

ISSN: $1596-5996$ (print); 1596-9827 (electronic)

(C) Pharmacotherapy Group, Faculty of Pharmacy, University of Benin, Benin City, 300001 Nigeria.

\title{
Spatholobus suberectus extract suppresses proliferation and EMT, and promotes apoptosis in palmitic acid induced vascular endothelial cells by inhibiting LncRNA MALAT1 via VEGF signaling pathway
}

\author{
Kebo Gu1, Lili He ${ }^{2 *}$ \\ ${ }^{1}$ Hematology Department, ${ }^{2}$ Hematology Department, Guang'anmen Hospital, China Academy of Chinese Medical Sciences, \\ Guang'anmen, China
}

*For correspondence: Email: Ilh1911@126.com; Tel: +86-13624836293

Sent for review: 23 Feb 2020

Revised accepted: 3 Mar 2020

\begin{abstract}
Purpose: In type 2 diabetes, palmitic acid could damage vessels and induce insulin resistance. This present in vitro study evaluates the possible role of Spatholobus suberectus (FSS) extract in diabetes. Methods: Human HUVECc cells were treated with palmitic acid, palmitic acid and Spatholobus suberectus extract. MALAT1 overexpression plasmid (pcDNA-MALAT1) and blank vector were transfected into the cells using lipofectamine 2000. RT-qPCR assay was used to evaluated the expression changes of IncRNA, VEGFR2 and VEGFA in the cells as well as Epithelial-Mesenchymal Transition (EMT) biomarkers and apoptosis. CCK-8 was used to detect cell viabilities of HUVECs. Expressions of proteins in VEGF signaling pathway were analyzed using Western Blot.

Results: LncRNA MALAT1 had high expression in diabetes-like cells and suppressed proliferation and EMT but promoted apoptosis. The SS extract promoted proliferation and EMT and repressed apoptosis in diabetes-like HUVECs cells. The promotion of apoptosis by LnCRNA MALAT1, inhibition of apoptosis and regulated functions of diabetes-like HUVECs cells by SS extract occurred via the VEGF signaling pathway

Conclusion: SS extract might contribute to survival of cells by inhibiting MALAT1 via VEGF signaling pathway in vitro, suggesting FSF might be a potential therapeutic agent in the treatment of diabetes.
\end{abstract}

Keywords: flavone of Spatholobus suberectus, diabetes, vascular endothelial cell, LncRNA MALAT1

\begin{abstract}
This is an Open Access article that uses a fund-ing model which does not charge readers or their institutions for access and distributed under the terms of the Creative Commons Attribution License (http://creativecommons.org/licenses/by/4.0) and the Budapest Open Access Initiative (http://www.budapestopenaccessinitiative.org/read), which permit unrestricted use, distribution, and reproduction in any medium, provided the original work is properly credited.
\end{abstract}

Tropical Journal of Pharmaceutical Research is indexed by Science Citation Index (SciSearch), Scopus, International Pharmaceutical Abstract, Chemical Abstracts, Embase, Index Copernicus, EBSCO, African Index Medicus, JournalSeek, Journal Citation Reports/Science Edition, Directory of Open Access Journals (DOAJ), African Journal Online, Bioline International, Open-J-Gate and Pharmacy Abstracts

\section{INTRODUCTION}

Diabetes is a metabolic disease with damaged functions of pancreatic islet or insulin resistance that may be caused by genetic factors, immune disorders, microbial infection or obesity, etc [1]. According to researches, there are about 0.387 billion people with diabetes all over the world and it is predicted by International Diabetes Federation (IDF) that the numbers will reach 0.592 billion by 2035 [2]. The high morbidity and mortality of cardiovascular diseases caused by diabetes has become a serious problem that threatens human health. Patients with diabetes 
often have abnormal angiogenesis, which is the main factor of diabetic cardiovascular complications [3]. Vascular endothelial growth factor (VEGF) plays an important role in regulating angiogenesis in physiological and pathological conditions [4]. VEGF and its receptor vascular endothelial factor receptor-2 (VEGFR-2) axis are affected by diabetes to militate growth of cardiovascular diseases [5]. In patients with type 2 diabetes, the level of palmitic acid is significantly increased, which could injure vascular endothelial cells and produce lipotoxicity. Moreover, it can influence to insulin signaling pathway, causing dysfunctions of vascular endothelial cells and leading to insulinresistant state [6].

LncRNA is a kind of RNA which has no proteincoding feature and full-length is longer than 200 nucleotides [11]. It is distributed in cytoplasm, nucleus and cell organelle but mainly in nucleus [12]. It has high tissue specificity, whose expressions are not only different in different tissues, but in same tissues; in different positions or different stages of the same tissue or organ $[13,14]$. LncRNA-MALAT1 has high expression in many cancer cell lines, which has close connection with cell migration and cancer recurrence [15]. For instance, MALAT1 could be an individual predictor for recurrence of liver cancer, suppression of which could inhibit survival rate and migration of HepG2 cells [16]. However, in diabetes MALAT1 is upregulated and its inhibition can decrease apoptosis of vascular endothelial cells.

Spatholobus suberectus (SS) Dunn is dried vine widely used in traditional medicine because it possesses pharmacological activities that include improvement of blood circulation, antiplatelet, anti-inflammation, anti-bacterial, neuroprotection, and anti-cancer effects $[6,17]$. The plant is known to contain many secondary compounds and flavonoids are the major bioactive substances, including 3',4',7-trihydroxyglavone, formononetin, calycosin, prunetin, eriodictyol, butin, liquiritigenin, plathymenin, dihydroquercetin and dihydrokaempferol as well as sterols, lignans, anthraquinones and glycosides $[7,8,18]$.

Among these compounds, flavones are the main active element as they have broad pharmacological functions including anti-tumor, antibiosis and control diseases of cardiovascular system, blood system, nervous system and atrophic arthritis [9]. S. suberectus has been reported to promote proliferation and differentiation of hemopoietic progenitor in mice to induce hematopoiesis[10]. Thus, there might be a connection between LncRNA MALAT1 and FSS because of the presence of flavones in FSS.

The objective of this study was to evaluate whether Spatholobus suberectus extract suppresses proliferation and EMT, and-promotes apoptosis of palmitic acid induced vascular endothelial cells by inhibiting LncRNA MALAT1 via VEGF signaling pathway using human HUVECs. In this study, palmitic acid was applied to induce vascular endothelial cells to build diabetes cellular model. Thereafter, FSS was used for intervention to detect proliferation of vascular endothelial cells and effects on VEGF signaling pathway. Proliferation and apoptosis of HUVECs and proteins in VEGF signaling pathway were analyzed for detecting mechanism of action.

\section{EXPERIMENTAL}

\section{Preparation of Spatholobus suberectus (SS)}

Dried SS was bought from a traditional Chinese drug store and pulverised into powder and a sample of $9.3 \mathrm{~g}$ was extracted with $50 \%$ ethyl alcohol in water at $50^{\circ} \mathrm{C}$ for $120 \mathrm{~min}$ twice. Then the extract was evaporated to dryness using a rotary evaporator (RE-52A, Shanghai Yarong Biochemistry, China) and the residue was made up to $100 \mathrm{ml}$ with $50 \%$ ethyl alcohol for further use.

\section{Cell culture and treatment}

Human HUVECs cells (purchased from ATCC, USA) were revived using standard procedure and then cultured in Dulbecco's Modified Eagle's Medium (DMEM) with $10 \%$ fetal bovine serum (FBS), $100 \mu \mathrm{g} / \mathrm{ml}$ penicillin and streptomycin at $37^{\circ} \mathrm{C}$ and $5 \% \mathrm{CO}_{2}$. Then, the cells in their log phase were digested with trypsin to make single cell suspension for further experiments which were shared into four parts;

Part 1: control (normal) cells (no SS and no palmitic acid treatment)

Part 2: diabetes-like cells - $600 \mu \mathrm{mol} / /$ palmitic acid was added so as to mimic the diabetes-like cells

Part 3: flavone treated diabetes-like cells 1 $600 \mu \mathrm{mol} / \mathrm{l}$ palmitic acid and $1.00 \mathrm{mg} / \mathrm{ml}$ of $\mathrm{SS}$ extract were added

Part 4: SS treated diabetes-like cells 2 $600 \mu \mathrm{mol} / \mathrm{l}$ palmitic acid and $2.00 \mathrm{mg} / \mathrm{ml}$ of $\mathrm{SS}$ extract were added 


\section{Transfection}

Adopting the method earlier described [19], each of the cells. types were seeded into 6-well plate and incubated in the DMEM medium until the confluence reached $70 \%$. The sequences of MALAT1 (Thermo Fisher Scientific, China) were amplified using polymerase chain reaction (PCR) and then subcloned into pcDNA3.1 (Thermo Fisher Scientific, China) to produce MALAT1 overexpression plasmid (pcDNA-MALAT1). The resultant pcDNA3.1-MALAT1 and blank vector were transfected into cells using lipofectamine 2000 (Thermo Fisher Scientific, China) according to the manufacturer's instruction. RT-qPCR was done to quantify the efficiency of transfection.

\section{RT-qPCR assay}

RT-qPCR evaluated the expression changes of IncRNA, VEGFR2 and VEGFA in normal cells and diabetes-like cells, overexpressed efficiency after transfection with PCDNA3.1-MALAT1 as well as in different treatment of SS extract in diabetes-like cells as previously described [19]. RT-qPCR also assessed EMT biomarkers Ecadherin, N-caderin and Vimentin mRNA expression in MALAT1 upregulated diabetes-like cells and normal disease cells, in different flavone treated disease cells and combined group with oe-MALAT1 and flavone treatment $(2 \mathrm{mg} / \mathrm{ml})$ so as to evaluate EMT functions in different groups. It was also used for apoptosis biomarker caspas-3 and anti-apoptosis biomarkers $\mathrm{Bcl}-2$ and $\mathrm{Bcl}-\mathrm{xl}$ in normal and disease cell lines, oe-MALAT1 and oe-NC groups, and different flavone treatment cell lines to investigate cell apoptosis. Cells in the groups mentioned were collected and RNAs were extracted strictly according to manufacture instructions using Trizol reagent (Thermo Fisher, USA). The total RNAs $(1 \mu \mathrm{g})$ were reversed to cDNAs in line with the specifications of the manufacturers of the Reverse Transcription reagent (Thermo Fisher, USA). Sequences of primers were: Bcl-2,forward:5' ACCAAGAAGCTGAGCGAGTGT-3', reverse,5'ACAAACATGGTCACGGTCTGC-3'; BclxL,forward:5'-GGAGCTGGTGGTTGACTTTCT3',reverse:5'-CCGGAAGAGTTCATTCACTAC3';GPADH,forward:5'GTCTCCTCTGACTTCAACAGCG-3', reverse,5'ACCACCCTGTTGCTGTAGCCAA-3'; IncRNAMALAT1, forward,5'GGCGGAATTGCTGGTAGTTT-3', reverse,5'AGCATAGCAGTACACGCCTT-3'.caspase3,forward:5'-CTGGACTGCGGTATTGAGAC3',reverse:5'-CCGGGTGCGGTAGAGTAAGC3'.VEGFR2,forward:5'-
GGACTCTCTCTGCCTACCTCAC-3',reverse:5GGCTCTTTCGCTTACTGTTCTG-3';

VEGFA,forward:

GGCAGCTTGAGTTAAACGAAC-3', reverse:5'ATGGATCCGTATCAGTCTTTCCTGG-3'.Ecadherin,Forward:5'-

TCTTCCAGGAACCTCTGTGATG-3'; Reverse:5'-CAATGCCGCCATCGCTTACACC-3'. Vimentin,forward:5'-

TACAGGAAGCTGCTGGAAGG-3', reverse:5'ACCAGAGGGAGTGAATCCAG-3';N-

cadherin,forward:5'-

ATTGGACCATCACTCGGCTTA-3', reverse: 5'CACACTGGCAAACCTTCACG-3' [19].

Conditions: predenaturation, $95{ }^{\circ} \mathrm{C}, 5 \mathrm{~min}$; denaturation, $95{ }^{\circ} \mathrm{C}, 30 \mathrm{~s}$; annealing, $55^{\circ} \mathrm{C}, 30 \mathrm{~s}$, extension, $72{ }^{\circ} \mathrm{C}, 30 \mathrm{~s}, 35$ cycles. Thereby, we evaluated the mentioned genes in the above groups with the cells transfected with PCDNA3.1MALAT1 or empty vector.

\section{CCK-8 assay}

Disease-like cells, extract treated disease-like cells $(1 \mathrm{mg} / \mathrm{ml}$ and $2 \mathrm{mg} / \mathrm{ml})$, and oe-MALAT1 extract treated cells $(2 \mathrm{mg} / \mathrm{ml})$ were selected for cell viability assay. Cells were seeded into 96well plate with $1 \times 10^{5}$ cells per well and then incubated in saturated humidity incubator at

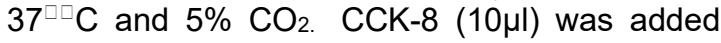
into each well after $24 \mathrm{~h}, 48 \mathrm{~h}$ and $72 \mathrm{~h}$ and the optical density (OD) values were determined at 490nm using microplate reader (Thermo Fisher Scientific, China).

\section{Western blot}

At $48 \mathrm{~h}$ after transfection, cells from the above groups were lysed using RIPA buffer (Thermo Fisher, USA) for $15 \mathrm{~min}$ on ice and then centrifuged to get supernatant liquid at 12000 rpm, $4{ }^{\circ} \mathrm{C}$ for $15 \mathrm{~min}$. Concentrations of proteins was evaluated using BCA protein assay kit (Thermo Fisher, USA) based on manufacturer's instruction to adjust proteins to the same concentrations. Proteins were denatured with Blue Juice ${ }^{\mathrm{TM}}$ Gel Loading Buffer (10x) (Invitrogen ${ }^{\mathrm{TM}}, \mathrm{USA}$ ) at $95^{\circ} \mathrm{C}$ for $8 \mathrm{~min}$ and then separated using SDS-PAGE and transferred into PVDF membranes. The membranes were then blocked with $8 \%$ skimmed milk powder and primary antibodies added prior to incubation at 4 ${ }^{\circ} \mathrm{C}$ overnight. The primary antibodies were: Ecadherin (1:1000; ab40772, Abcam, Cambridge, UK), N-cadherin (1:1000; ab18203), Vimentin $(1: 1000 ;$ ab8069). VEGF signaling proteins, VEGF-A (1:100; ab52917), VEGFR-2 (1:1000; ab39256) and GAPDH (1:2000; ab181602). For apoptosis-related proteins, the primary 
antibodies from Beyotime (Shanghai, China) were: $\mathrm{Bcl}-2(1: 1000, \quad \mathrm{AB} 112), \quad \mathrm{Bcl}-\mathrm{xl}(1: 1000$, $A B 126)$ and Caspas-3(1:2000, AF1213). Then, goat anti-rabbit IgG H\&L (HRP) (1:800; ab205718) and goat anti-mouse IgG H\&L (HRP) (1:800; ab205719) were added for incubation at $37^{\circ} \mathrm{C}$ after $1 \mathrm{~h}$. Next, Pierce ${ }^{\mathrm{TM}} \mathrm{ECL}$ Plus Western Blotting Substrate (Thermo Scientific ${ }^{\mathrm{TM}}$, USA) was applied for coloration and gray color of proteins were used to quantify expressions of proteins.

\section{Statistical analysis}

Each experiment was done in triplicates, analysed using SPSS 19.0 (IBM, USA) and data were reported as mean \pm SD. Comparison of data was achieved using Student's $T$ test as appropriate. $\mathrm{P}<0.05$ was considered significant at $95 \%$ confidence interval.

\section{RESULTS}

LncRNA MALAT1 had high expression in
diabetes-like cells and $\begin{array}{r}\text { suppressed } \\ \text { proliferation and EMT but promoted } \\ \text { apoptosis }\end{array}$

When the expressions of IncRNA MALAT1 was evaluated in normal human HUVECs and palmitic acid induced HUVECs, IncRNA MALAT1 expressed higher in diabetes-like HUVECs (Figure 1A) when compared to normal cells. After MALAT1 was overexpressed, its expression was higher than control group in induced cells (Figure 1B). The results of CCK-8 showed that overexpressed IncRNA MALAT1 significantly reduced cell viability of induced HUVECs (Figure 1C). As for EMT, analysis of RNA expression of biomarkers showed that E-cadherin was promoted while $\mathrm{N}$-cadherin and Vimentin were inhibited by up-regulated IncRNA MALAT1 (Figure 1D-F). Moreover, analysis of the RNA expression of biomarkers related to apoptosis showed that in overexpressed MALAT1 group, expressions of $\mathrm{Bcl}-2$ and $\mathrm{Bcl}-\mathrm{xL}$ was suppressed while expressions of caspase- 3 was promoted (Figure 1G-I).

\section{Spatholobus suberectus extract promoted proliferation and EMT and repressed apoptosis in diabetes-like HUVECs}

With increasing concentrations of $\mathrm{S}$. suberectus extract $(0.00 \mathrm{mg} / \mathrm{ml}, 1.00 \mathrm{mg} / \mathrm{ml}$ and $2.00 \mathrm{mg} / \mathrm{ml}$ corresponding to increasing concentration of flavones), viability of HUVECs was increasingly up-regulated (Figure 2A). E-cadherin expression was significantly decreased and expressions of $\mathrm{N}$-cadherin and Vimentin was increased as densities of flavones of $S$. suberectus upregulated (Figure 2B-D). RNA expression of apoptosis proteins indicated that expressions of $\mathrm{Bcl}-2$ and $\mathrm{Bcl}-\mathrm{xL}$ was increased but caspase-3 was repressed with increasing concentrations of flavone (Figure 2E-G).
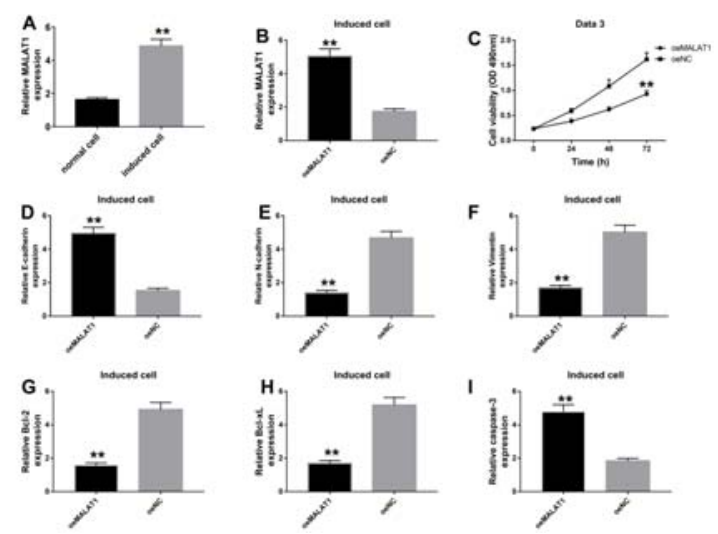

Figure 1: IncRNA MALAT1 expressed higher in induced and suppressed proliferation and EMT with promoting apoptosis. A, B. RT-qPCR was applied to validated expressions of IncRNA MALAT1 and overexpressed IncRNA MALAT1, $P<0.05$. C. CCK-8 was used to detect cell viabilities, $P<0.05$. D, E. Expressions of factors in EMT and apoptosis were measured with RT-qPCR, $\mathrm{P}<0.05$

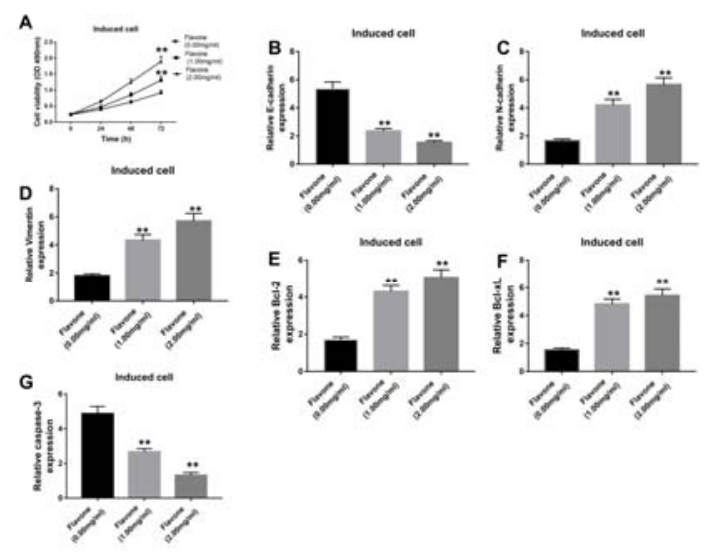

Figure 2: Spatholobus suberectus extract upregulated proliferation and EMT and suppressed apoptosis. A, In the presence of $S$. suberectus extract, cell viability was detected by CCK-8, B, Expressions of RNAs in EMT was validated using RT-qPCR; C, Apoptosis was measured through RT-qPCR

LncRNA MALAT1 promoted the disease cell apoptosis through VEGF signaling pathway

In normal human HUVECs and palmitic acid induced human HUVECs, VEGF-A and VEGFR2 were detected for RNA expression, which showed that VEGF-A and VEGFR-2 were activated in diabetes-like HUVECs compared to 
normal HUVECs (Figure 3A-B). RT-qPCR and Western Blot results indicated that up-regulated IncRNA MALAT1 activated VEGF-A and VEGFR2 in induced cells (Figure 3C-D). When VEGFR2 suppressor SKLB610 was added, expression of VEGF-A and VEGFR-2 was significantly suppressed (Figure 3E-F). Anti-apoptosis biomarkers $\mathrm{Bcl}-2$ and $\mathrm{Bcl}-\mathrm{xL}$ were down regulated while caspas-3 was increased by upregulated MALAT1, implying that cell apoptosis was increased by MALAT1 upregulation. However, when SKLB610 was applied together with overexpressed MALAT1, the effects were counteracted in part with increased anti-apoptosis biomarkers and decreased Caspas-3 compared to oe-MALAT1 group, suggesting that MALAT1 might promote cell apoptosis via VEGF signaling pathway (Figure 3G-H).
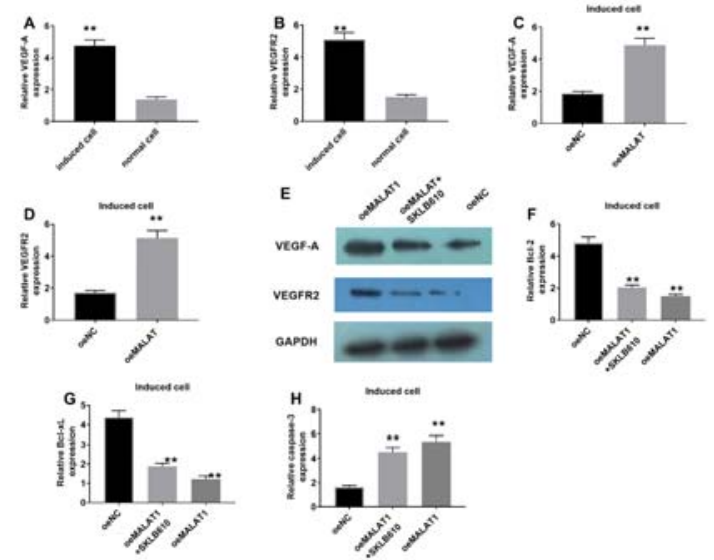

Figure 3: IncRNA MALAT promoted apoptosis via VEGF signaling pathway. A. RT-qPCR was applied to detect expressions of RNAs related to proteins in VEGF signaling pathway, $P<0.05$. B. Expressions of factors in VEGF signaling pathway with overexpressed IncRNA MALAT1 were evaluated using RT-qPCR, $P<0.05$. C. Western blot was for measuring levels of proteins in VEGF signaling pathway, $P<0.05$. $D$. Expressions of RNAs related to apoptosis after VEGF signaling pathway was suppressed with SKLB610 were measured through RT-qPCR, $P<0.05$

\section{Spatholobus suberectus extract inhibits MALAT1 and regulated functions of HUVECs via VEGF signaling pathway}

In palmitic acid induced human HUVECs, protein expression of VEGF-A and VEGFR-2were detected by Western Blot (Figure 4A). With increasing concentrations of $S$. suberectus extract, VEGF-A and VEGFR-2 expression was both inhibited in a dose-dependent way. Thereafter, IncRNA MALAT1 expression was significantly lower after $\mathrm{S}$. suberectus extract was added into human HUVECs in a dose-dependent manner (Figure 4B). Moreover, biomarkers related to EMT were detected as well; relative RNA expression of E-cadherin was promoted by overexpression of MALAT1 but the extract inhibited the function of overexpressed MALAT1 (Figure 4C). Meanwhile, suppressed N-cadherin and Vimentin by up-regulated IncRNA MALAT1 was reversed in the presence of the extract (Figure 4D-E). Similar results were obtained in apoptosis analysis. Inhibited expression of $\mathrm{Bcl}-2$ and $\mathrm{Bcl}-\mathrm{xL}$ by overexpressed IncRNA MALAT1 was up-regulated by the extract in a dosagedependent manner. The caspase- 3 , which was activated in overexpressed IncRNA MALAT1 groups, was inhibited by the extract as well (Figure 4F).

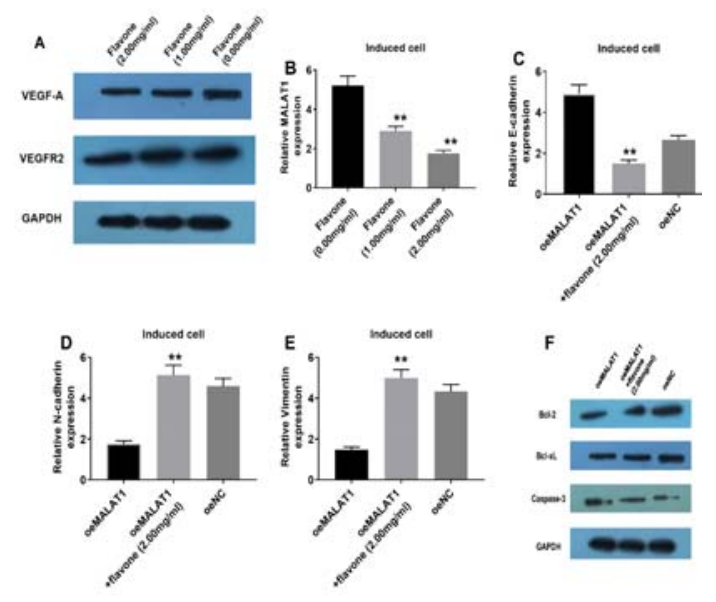

Figure 4: Flavone of Spatholobus suberectus regulate functions of cells and inhibit IncRNA. A, Western blot was applied to measure expressions for proteins; B, After the extract of Spatholobus suberectus was added, IncRNA MALAT1 expressions were detected through RT-qPCR; C, Factors related to EMT were evaluated through RT-qPCR; D, Western blot was used for validating expressions of proteins in VEGF signaling pathway.

\section{DISCUSSION}

Based on earlier study, catechinic acid of flavone present in $\mathrm{S}$. suberectus may induce activation of GM-CSA, BPA, MK-CSA to increase creation of blood cells and also promote red blood cells and hemoglobin of mice with anemia [20]. LncRNA MALAT1 show higher expression in patients with various complications related to diabetes and it promoted apoptosis in vitro $[20,21]$. This study has revealed that LncRNA MALAT1 had high expression in diabetes-like cells and suppressed proliferation and EMT but promoted apoptosis. The SS extract promoted proliferation and EMT and suppressed apoptosis but increased cell viability in diabetes-like HUVECs cells. Using RTqPCR and Western Blot, high expression of 
VEGFR-2 and VEGF-A in disease-like cells and oe-MALAT1 activated VEGF signaling. With Inactivation of VEGF signaling with the antagonist of VEGF pathway, SKLB610, reduced the cell death indicating that MALAT1 may promote cell death via VEGF signaling pathway.

By connecting SS extract with VEGF and MALAT1 using different dosages of SS extract, it became clear that the proteins in VEGF signaling, VEGFR-2 and VEGF-A, gradually decreased as the dosage of SS extract increased. EMT process and apoptosis were examined and the findings pointed out that overexpressed MALAT1 inhibited the EMT process and promoted apoptosis while SS extract could offset the apoptosis-promoting effect of MALAT1 in vitro, implying that SS extract might protect against cell apoptosis through MALAT1. In conclusion, it was suggested that SS extract might protect the human vascular endothelial cells from injury induced by palmitic acid through inhibiting MALAT1 via VEGF signaling pathway. VEGF was first discovered in ascites and tumor tissues of guinea pig in 1983 [22]. There are five members in VEGF signaling pathway: VEGF-A, VEGF-B, VEGF-C, VEGF-D and placental growth factor (PIGF). VEGFR-2 (KDR/Flk-1) is the primary function receptor which is mainly expressed in vascular endothelial cells [4].It was reported previously that VEGF signaling pathway was activated in glucose-induced HUVECs [23].

\section{CONCLUSION}

LncRNA MALAT1 had higher expression in palmitic acid induced HUVECs and regulated cell functions via VEGF signaling. The flavones present in $S$. suberectus could promote cell viability, EMT and inhibit apoptosis by inhibiting MALAT1 via VEGF signaling pathway suggesting that SS EXTRACT might be a potential remedy for injuries involving blood vessel in diabetes.

\section{DECLARATIONS}

\section{Conflict of interest}

No conflict of interest is associated with this work.

\section{Contribution of authors}

We declare that this work was done by the authors named in this article and all liabilities pertaining to claims relating to the content of this article will be borne by the authors.

\section{Open Access}

This is an Open Access article that uses a funding model which does not charge readers or their institutions for access and distributed under the terms of the Creative Commons Attribution License (http://creativecommons.org/licenses/by/ 4.0) and the Budapest Open Access Initiative (http://www.budapestopenaccessinitiative.org/rea d), which permit unrestricted use, distribution, and reproduction in any medium, provided the original work is properly credited.

\section{REFERENCES}

1. Tao Z, Shi A, Zhao J. Epidemiological Perspectives of Diabetes. Cell Biochem Biophys, 2015; 73(1): 181-5.

2. Yang $W$. Changing characteristics of the type 2 diabetes epidemic of China and other Asian countries. J Diabetes Investig, 2013; 4(3): 223-4.

3. Kolluru GK, Bir SC, Kevil CG. Endothelial dysfunction and diabetes: effects on angiogenesis, vascular remodeling, and wound healing. Int J Vasc Med, 2012; 2012: 918267.

4. Shibuya M. VEGFR and type-V RTK activation and signaling. Cold Spring Harb Perspect Biol, 2013; 5(10): a009092.

5. Waltenberger J. VEGF resistance as a molecular basis to explain the angiogenesis paradox in diabetes mellitus. Biochem Soc Trans, 2009; 37(Pt 6): 1167-70.

6. Suiter $C$ et al. Free Fatty Acids: Circulating Contributors of Metabolic Syndrome. Cardiovasc Hematol Agents Med Chem, 2018; 16(1): 20-34.

7. Zhao P, Alam MB, Lee $S$ et al. Spatholobus suberectus Exhibits Antidiabetic Activity In Vitro and In Vivo through Activation of AKT-AMPK Pathway. Evid Based Complement Alternat Med, 2017; 2017: 6091923.

8. Chen S, Wang A, Lin L, Qiu H, Wang Y, Wang Y. In Vitro Study on Anti-Hepatitis C Virus Activity of Spatholobus suberectus Dunn. Molecules, 2016. 21(10): 1367.

9. Chen HL, Yang J, FU YF, Meng XN, Zhao WD, Hu TJ. Effect of total flavonoids of Spatholobus suberectus Dunn on PCV2 induced oxidative stress in RAW264.7 cells. BMC Complement Altern Med, 2017; 17(1): 244.

10. Lee $B J$, Jo $I Y, B u \quad Y$ et al. Antiplatelet effects of Spatholobus suberectus via inhibition of the glycoprotein IIb/IIla receptor. J Ethnopharmacol, 2011; 134(2): 460-7.

11. Qiu MT, Hu JW, Yin R, Xu L. Long noncoding RNA: an emerging paradigm of cancer research. Tumour Biol, 2013; 34(2): 613-20.

12. Djebali S, Davis CA, Merkel A et al. Landscape of transcription in human cells. Nature, 2012; 489(7414): 101-8.

13. Zhao $W, M u Y, M a L$ et al. Systematic identification and characterization of long intergenic non-coding RNAs in fetal porcine skeletal muscle development. Sci Rep, 2015; 5: 8957. 
14. Han L, Zhang $K$, Shi Z, Zhang J, et al. LncRNA pro fi le of glioblastoma reveals the potential role of IncRNAs in contributing to glioblastoma pathogenesis. Int $\mathrm{J}$ Oncol, 2012; 40(6): 2004-12.

15. Yang $F$, Yi F, Han X, Du Q, Liang Z. MALAT-1 interacts with hnRNP C in cell cycle regulation. FEBS Lett, 2013; 587(19): 3175-81.

16. Lai MC, Yang Z, Zhou L, Zhu QQ, Xie HY, Zhang F, Wu $L M$, Chen $L M$, Zheng SS. Long non-coding RNA MALAT-1 overexpression predicts tumor recurrence of hepatocellular carcinoma after liver transplantation. Med Oncol, 2012; 29(3): 1810-6.

17. Zhang Y, Guo L, Duan L, Dong X, Zhou P, Liu EH, Li P. Simultaneous determination of 16 phenolic constituents in Spatholobi Caulis by high performance liquid chromatography/electrospray ionization triple quadrupole mass spectrometry. J Pharm Biomed Anal. 2015; 102:110-8.

18. Jiang SY, Huang K, Liu W, Fu FM, Xu J. Combined Autodock and comparative molecular field analysis study on predicting 5-lipoxygenase inhibitory activity of flavonoids isolated from Spatholobus suberectus Dunn. Zeitschrift Fur Naturforschung C Journal of Biosciences. 2015;70(3-4):103-13.
19. Chang J, Xu W, Du X, Hou J. MALATI silencing suppresses prostate cancer progression by upregulating miR-I and downregulating KRAS. OncoTargets Ther. 2018; 11:3461-3473.

20. Wang DX1, Liu P, Chen YH, Chen RY, Guo DH, Ren $H Y$, Chen ML. Stimulating effect of catechin, an active component of Spatholobus suberectus Dunn, on bioactivity of hematopoietic growth factor. Chin Med J (Engl), 2008. 121(8):752-5.

21. Liu JY, Yao J, Li XM, Song YC, Wang XQ, Li YJ, Yan B, Jiang $Q$. Pathogenic role of IncRNA-MALAT1 in endothelial cell dysfunction in diabetes mellitus. Cell Death Dis, 2014. 5: e1506.

22. Senger DR, Galli SJ, Dvorak AM, Perruzzi CA, Harvey VS, Dvorak HF. Tumor cells secrete a vascular permeability factor that promotes accumulation of ascites fluid. Science, 1983. 219(4587): 983-5.

23. $X u L$, Li Y, Yin L, Qi Y, Sun $H$, Sun $P, X u M$, Tang $Z$, Peng. miR-125a-5p ameliorates hepatic glycolipid metabolism disorder in type 2 diabetes mellitus through targeting of STAT3. Theranostics, 2018. 8(20):55935609. 